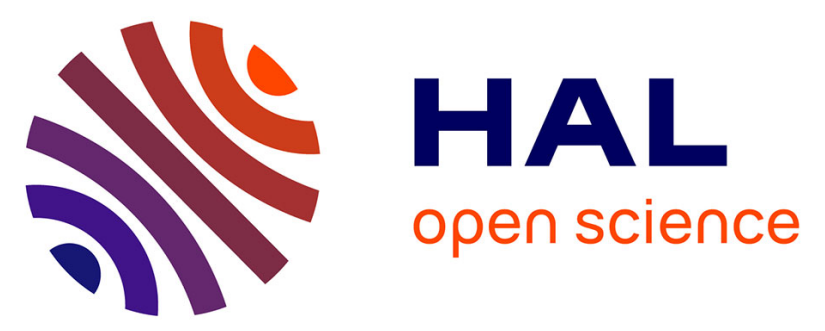

\title{
Corpus des marques lyonnaises sur sigillée et imitations de sigillée [L'atelier de la Muette]
}

\author{
Martine Genin, Jacques Lasfargues, Maurice Picon
}

\section{To cite this version:}

Martine Genin, Jacques Lasfargues, Maurice Picon. Corpus des marques lyonnaises sur sigillée et imitations de sigillée [L'atelier de la Muette]. Gallia - Archéologie de la France antique, 1996, Les productions des ateliers de potiers antiques de Lyon (1ère partie) : les ateliers précoces, 53, pp.193213. 10.3406/galia.1996.3202 . hal-01923629

\section{HAL Id: hal-01923629 \\ https://hal.science/hal-01923629}

Submitted on 7 Feb 2020

HAL is a multi-disciplinary open access archive for the deposit and dissemination of scientific research documents, whether they are published or not. The documents may come from teaching and research institutions in France or abroad, or from public or private research centers.
L'archive ouverte pluridisciplinaire HAL, est destinée au dépôt et à la diffusion de documents scientifiques de niveau recherche, publiés ou non, émanant des établissements d'enseignement et de recherche français ou étrangers, des laboratoires publics ou privés.

\section{(ㅇ)(1) $\$$}

Distributed under a Creative Commons Attribution - NonCommercial - NoDerivatives| 4.0 


\title{
Corpus des marques lyonnaises sur sigillée et imitations de sigillée
}

\author{
Martine Genin, Jacques LASfargues et Maurice PiCON
}

\section{LES MARQUES LYONNAISES SUR SIGILLÉE (tabl. X à XII)}

Présentation des notices et figures du catalogue : dessins à l'échelle $1 / 1$.

Les marques ont été classées dans l'ordre du Corpus ysorum Arretinorum (CVA) ou, à défaut, dans l'ordre Ho.jhabétique. Lorsqu'une estampille comporte deux noms, elle est indiquée au nom de celui qui est considéré tharlitionnellement comme le patron.

Provenances :

ateliers de la Muette $=$ fouilles de 1966 et 1975 (Lasfargues, 1976) ;

- musée f.a. = musée fonds ancien : Citerne R.R., découvertes isolées à Lyon - Trion, Minimes - ou en dehors de Lyon ;

- camp militaire de Haltern (von Schnurbein, 1982);

- le sigle MML suivi d'un numéro renvoie à la série d'analyses faite sur les timbres de l'atelier;

- le sigle EAD suivi d'un numéro renvoie à la série d'analyses faite sur le fonds ancien du MCGRL;

- le sigle HAL, suivi d'un numéro renvoie à la série d'analyses faite sur les timbres de Haltern.

Description sommaire de la marque : forme de l'empreinte, présence ou absence d'encadrement, caractéristiques des lettres (ligatures, etc.). Les dimensions sont données en millimètres.

Formes sur lesquelles l'estampille a été relevée : ceci ne signifie pas que d'autres formes portant cette marque soient à exclure; elles n'y ont pas encore été attestées. Mention de la fréquence des cas où l'estampille est rencontrée sur telle ou telle forme, excepté pour certaines marques ou graphies publiées en 1976 et dont ne figurait aucun exemplaire dans les dix contextes étudiés en vue de cette publication. Les résultats détaillés de l'étude des fréquences des marques et des graphies par formes figurent dans le chapitre consacré aux timbres (cf. supra, p. 55-63).

Renvoi à l'ouvrage de A. Oxé et H. Comfort, Corpus Vasomum Arretinorum, Bonn, 1968 est indiqué par l'abréviation O.C. La mention " non reproduite dans O.C. " signifie que l'estampille peut figurer dans le répertoire de O.C., mais sans y avoir été reproduite.

\section{ACASTVS : O.C. 8}

1. ACAST (pl. 89, 2-3)

Muette 66 (MML 105). Marque dégradée peu lisible. Empreinte rectangulaire et irrégulière ( 15 x 6 env.). Sans encadrement.

Sur plateau. Unique. O.C. 8 a, b, c.

2. ACA

Haltern, $n^{\circ}$ 2-3 (HAL 2 et 243). Empreinte rectangulaire $(11 \times 9)$. Encadrement.

$\mathrm{n}^{\circ} 2$ : assiette NI, $\mathrm{n}^{\circ} 3:$ assiette du service IC.

3. ACA (pl. 89, 1)

Musée f.a. (EAD 69). Empreinte rectangulaire (14 x 6). Sans encadrement.

Sur assiette NI.

4. (...) ASV (pl. 89, 4)

Haltern, $n^{\circ} 16$ (HAL 244). Empreinte rectangulaire (? x 6). Encadrement.

Sur bol du service II. 


\begin{tabular}{|c|c|c|}
\hline marques & O.C. $n^{\circ}$ & références \\
\hline ACASTVS & 8 & Muette 1976, 1, I. Haltern 1982, no 2.3.16. Musée f.a., EAD 66. \\
\hline ALBANVS & 36 & Muette 1976, II, 1. \\
\hline ALPVS & $?$ & Muette 1976, III, 1. \\
\hline ANNIVS & 83 & Muette 1976, IV, 1 \\
\hline ATEIVS & $144-145$ & Haltern 1982. \\
\hline EVHODVS & 161 & Musée f.a., EAD 26. \\
\hline XANTHVS & 177 & Muette 1976, XXXIX, 1-2.Lyon citerne R.R., EAD 8. Haltern 1982, n॰421-422. \\
\hline ZOILVS & 186 & Muette 1966, XL, 1, MML 46. \\
\hline C.ATTIVS & 205 et 212 & Muette 1976, VI, 1 à 10 et VI, 11. Haltern 1982, n445-446 et n $464-465$. \\
\hline AVITIIVS & $?$ & Muette 1976, VIII, 1. \\
\hline BASSVS & 323 & Lyon citerne R.R., EAD 7. \\
\hline L.BRINNIVS & 335 & Musée f.a., EAD 44. \\
\hline CALAMVS & 359 & Muette $1976,1 X, 1$ \\
\hline CRESTVS & 425 & Muette $1976, X, 1$ \\
\hline CLARVS & $443-444$ & Musée f.a., EAD 69. Haltern 1982, n489-490, nº 495. \\
\hline CLARI ATEIANI & 446 & Haltern $1982, n^{\circ} 497$. \\
\hline $\mathrm{COCl}$ & $?$ & Haltern $1982, n \circ 870$ \\
\hline COR & 467 & Musée f.a. (Lyon, Trion), EAD 38. \\
\hline DIOMEDES & 599 & Musée f.a., EAD 49. Haltern 1982, n511, n०518. \\
\hline C.FASTIDIENVS & 672 & Muette $1976, \mathrm{XI}, 1$ \\
\hline FAVSTVS & 679 & Muette 1976, XII, 1. \\
\hline FELLIX & $682,684,685$ & Musée f.a., EAD 50. Haltern 1982, n॰526, n॰528. \\
\hline FIRMVS & 695 & Muette 1976, XIII, 1-2. \\
\hline FONTEIANVS & 703 & Muette 1976, XV, 1. Haltern 1982, n॰532, n॰544, n॰549.Musée f.a., EAD 45. \\
\hline FRONTO & 713 & Muette 1976, XVI, 1 à 6. \\
\hline GAMVS & 725 & Muette 1976, XVII, 1 à 3. \\
\hline L.SEMPRONIVS et L.GELLIVS & 747 & Musée f.a., EAD 21. Haltern 1982, n॰568, n॰570. \\
\hline HA & ? & Muette 1976, XVIII, 1. \\
\hline INACHVS & 827 & Muette $1976, \mathrm{XX}, 1$. \\
\hline ICE ou ICV & ? & Muette 1976, XXI. \\
\hline IVCVNDVS & $835 ?$ & Musée f.a., EAD 64. \\
\hline LESBIVS & ? & Muette 1976, XXII, 1. \\
\hline TITVS MALIVS FORTVNATVS & 942 & Muette 1976, XXIII, 1-2. \\
\hline (N.NAEVIVS) HILARVS & 1084 & Muette 1976, XIX, 1-2. \\
\hline NIGER & $1123 ?$ & Wuette 1976, XXIV, 1. \\
\hline (M.PVPIVS) FLOS () F (ECIT?) & 1204 & Muette 1976, XIV, 1-2. \\
\hline QVADRATVS & $1460-1461$ & Muette 1976, XXV, 1-2. \\
\hline QVARTIO & 1463 & Muette 1976, XXVI, 1. \\
\hline RASINIVS & 1485 et 1487 & Muette 1976, XXVII, 1 à 7 . Haltern 1982, n 676. \\
\hline RVSTICVS & 1619 & Muette 1976, XXVIII, 1-2. \\
\hline SABINVS & $1645 ?$ & Musée f.a., EAD 57. \\
\hline C.SENTIVS & $1730-1732$ & Muette 1976, XXIX, 1 à 16. Haltern 1982, no 714. \\
\hline STABILIO & 1847 & Muette 1976, XXX, 1. \\
\hline TERTIVS & $1945 ?$ & Muette 1976, XXXI, 1. \\
\hline TEVC & $?$ & Muette 1976, XXXII, 1. \\
\hline L.THYRSVS & $831,2062 / 68$ & Muette 1976, XXXIII, 1 à 20. \\
\hline TIGRANVS & 1995 & Muette 1976, XXXIV, 1 à 6. \\
\hline TVLLIIVS & $?$ & Muette 1976, XXXV, 1-2. \\
\hline SEX.VARIVS & $2235-2237$ & Muette 1976, XXXVIII, 1 à 3. Haltern 1982, n`857-858. Musée f.a., EAD 56. \\
\hline VILLIVS & 2372 & Lyon citerne R.R., EAD 9. Musée f.a., EAD 55. \\
\hline VMBRICIVS & 2385 & Muette 1976, XXXVI, 1-2. \\
\hline VRBANVS & 2498 & Muette 1976, XXXVII, 1. Haltern 1982, n॰862-863. \\
\hline AMA? & $?$ & Haltern 1982, n॰902. \\
\hline ITVTN & ? & Haltern 1982, n॰878. \\
\hline REI? & ? & Haltern $1982, n \cap 879$. \\
\hline TIT.ROR & ? & Musée f.a., EAD 48. \\
\hline (...) TIF & $?$ & Haltern $1982, n^{\circ} 873$ \\
\hline VLON ou ELON & $?$ & Muette 1966, XLI,1. \\
\hline anépigraphes & ? & Muette 1966, XLII, 1-2, MML 103. \\
\hline de lecture conjecturale & ? & Muette 1966 et 1975, MML $101,111,184$. \\
\hline
\end{tabular}

Tabl. X. I.iste générale des marques lyonnaises sur céramique sigillée. 
Tabl. XI. Liste des marques lyonnaises non attestées sur le site de l'atelier de la Muette.

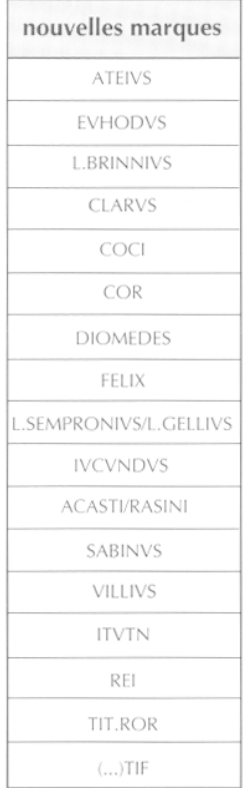

Tabl. XII. Liste des graphies non attestées sur le site de l'atelier de la Muetle.

\begin{tabular}{|c|}
\hline nouvelles graphies \\
\hline ACASTVS \\
\hline ALBANVS \\
\hline XANTHVS \\
\hline FONTEIANVS \\
\hline C.SENTIVS \\
\hline SEX.VARIVS \\
\hline VRBANVS \\
\hline
\end{tabular}

\section{ALBANVS : O.C. 36}

\section{ALBAN}

Muette 66 (MML 104). Empreinte rectangulaire (14 x 7). Encadrement peu net. Ligature $A N$.

Sur bol du service II. Unique. O.C. 36 (n) 1.

\section{ALBAN}

Muette 66 (MML, 42). Empreinte rectangulaire très nette $(13 \times 6)$. Sans encadrement. Ligatures AL et AN. Sur bol du service I. Unique. -O.C. 36 K2.
3. ALBA (pl. 89, 5)

Haltern, n* 27-28 (HAI 1 et 245). Fmpreintes rectangulaires $(12 \times 5 ; 13 \times 7)$. Encadrements.

Sur bols du service II.

\section{ALPVS : non répertoriée dans O.C.}

\section{ALPI}

Muette 66 (MML 84). Empreinte vaguement rectangulaire $(10 \times 5)$. Sans encadrement. Ligature AI.P.

Marque apparaissant exclusivement sur des bols, en majorité du service II ( I seul ex. du service I).

\section{ANNIVS : O.C. 83}

1. CERDO/C. ANNI Muette II (MML 41). Empreinte nettement ovalisée ( $12 \times 6)$. Sans encadrement.

Sur bol. Unique.

\section{ATEIVS : O.C. 144}

1. ATEI (pl. 89, 6-9)

Haltern $\mathrm{n}^{\circ}$ 82, 84, 85, 87 (HAL, 25-248-249-26). Empreintes rectangulaires $(13 \times 7 ; 12 \times 7 ; ? \times 7 ; 13 \times$ 8). Sans encadrements.

Sur bols du service II.

2. ATEI (pl. 89, 10)

Haltern $n^{\circ} 89$ (HAI . 27). Empreinte rectangulaire (? $\mathrm{x}$ $4,5)$. Sans encadrement.

Sur bol (service I?).

3. A (T) EI (pl. 89, 11-13)

Haltern $n^{\circ}$ 143-144-145 (HAL 253, 252, 45). Empreintes rectangulaires $(12,1 \times 7 ; 11,5 \times 7 ; 11 \times 7)$. Sans encadrements. Ligature AT.

$n^{\circ} 143$ sur bol NI, n $144-145$ sur bols du service II.

4. A (T) EI (pl. 89, l4)

Haltern $n^{\circ} 151$ (HAL 47). Empreinte rectangulaire (? x 5). Sans encadrement. Ligature TE.

Sur bol du service II. 
5. A (T) EII (pl. 89, 15)

Haltern $n^{\circ} 173$ (HAL 48). Empreinte rectangulaire $(15 \times 7,5)$. Sans encadrement. Ligature AT.

Sur bol du service I (?).

6. A (T) EI (pl. 89, 16-17)

Haltern ${ }^{\circ}$ 174-175 (HAL 49-50). Empreintes rectangulaires $(12 \times 6,5 ; 13 \times 6,5)$. Encadrement. Ligature AT.

$\mathrm{n}^{\circ} 174$ sur bol du service I (?), $\mathrm{n}^{\circ} 175$ sur bol Ha. 8 .

7. A (T) EI (pl. 89, 18)

Haltern $n^{\circ} 187$ (HAL 54). Empreinte rectangulaire $(9,5 \times 6)$. Sans encadrement. Ligature TE?

Sur bol du service II.

8. AEI (pl. 89, 19)

Haltern $n^{\circ} 188$ (HAL 55). Empreinte rectangulaire $(9 \times 15)$. Sans encadrement.

Sur bol du service II.

9. AEI palme (pl. 89, 20-22)

Haltern $\mathrm{n}^{\circ}$ 189, 191, 192 (HAL 56, 254, 58).

Empreintes rectangulaires $(13 \times 6 ; 14 \times 6 ; 14 \times 6)$.

Sans encadrement.

Ligatures AT.

$\mathrm{n}^{\circ} 189$ sur assiette (service I?), $\mathrm{n}^{\circ} 191$ sur assiette du service IC, $n^{\circ} 192$ sur assiette du service II.

10.A (T) EII (pl. 89, 23)

Haltern $n^{\circ} 195$ (HAL 57). Empreinte rectangulaire $(15 \times 8)$. Sans encadrement.

Sur bol du service $\mathrm{I}$.

11.A (T) EI palme (pl. 89, 24)

Haltern $n^{\circ} 203$ (HAL 59). Empreinte rectangulaire (15 x 8). Sans encadrement. Ligature AT.

Sur bol du service II.

12. A (T) EI palme (pl. 89, 25)

Haltern $n^{\circ} 205$ (HAL 60). Empreinte rectangulaire $(16,5 \times 9)$. Sans encadrement. Ligature AT.

Sur bol (service I?).

13. AEI palme (pl. 89, 26)

Haltern $n^{\circ} 208$ (HAL 61). Empreinte rectangulaire $(11 \times 5,5)$. Sans encadrement. Ligature AT.

Sur bol du service $\mathrm{I}$.
14. A (T) EI (pl. 89, 27)

Haltern $n^{\circ} 228$ (HAL 67). Empreinte rectangulaire (? x 7). Sans encadrement. Ligature AT.

Sur bol.

15. A (T) EI palme (pl. 89, 28-29)

Haltern $n^{\circ}$ 236, 239 (HAL 71, 72). Empreintes rectangulaires à angles arrondis (env. $12 \times 6 ; 12 \times 4,5$ ). Sans encadrement. Ligatures TE.

$n^{\circ} 236$ et 239 sur assiettes NI.

16. IA (T) EI (pl. 89, 30-31)

Haltern $\mathrm{n}^{\circ}$ 242, 243, 245 (HAL 73, 308, 307). Empreintes rectangulaires (? $\times 4,5 ; 13 \times 5 ; 11 \times 4)$. Sans encadrement. Ligatures TE.

Sur bols du service II.

17. (ATE) I palme (pl. 89, 32)

Haltern $n^{\circ} 281$ (HAL 80). Empreinte rectangulaire (11 ? x 5). Sans encadrement. Ligature ATE.

Sur bol (Ha. 11 ou Ha. 15?).

18. ATEI palme rétrograde (pl. 89, 33-34)

Haltern $n^{\circ}$ 284-285-286 (HAL 255-256-257). Empreintes rectangulaires $(12 \times 8 ; 12 \times 8 ; 12 \times 8)$. Encadrement haut/bas.

$\mathrm{n}^{\circ} 284$ et 286 sur assiettes du service II, $\mathrm{n}^{\circ} 285$ sur FNI.

19. AEII (pl. 89, 35)

Haltern $n^{\circ} 291$ (HAL 81). Empreinte rectangulaire $(13 \times 4,5)$. Sans encadrement. Ligature TE.

Sur bol du service II.

20. ATI (pl. 89, 36)

Haltern $n^{\circ} 295$ (HAL 85). Empreinte rectangulaire (13 x 6). Sans encadrement.

Sur assiette du service IC.

\section{C.N. ATEIVS : O.C. 145}

1. (C) N A (T) EI (pl. 89, 37)

Haltern $n^{\circ} 309$ (HAL 87). Empreinte rectangulaire (12 x 8). Sans encadrement. Ligature AT.

Sur assiette NI. 


\section{EVHODVS : O.C. 161}

1. HEVODI (pl. 90, 1)

Musée f.a. (EAD 26). Empreinte rectangulaire (13 x 5). Sans encadrement.

Sur assiette du service II.

\section{XANTHVS : O.C. 177}

\section{XANTVS}

Muette 66 (MML. 98). Empreinte rectangulaire (14 x $4,5)$. Sans encadrement.

Sur assiette. Unique. Non répertoriée dans O.C. 177.

2. XANTI (pl. 90, 2)

Musée f.a., citerne R.R. (EAD 8). Empreinte rectangulaire $(11 \times 4,5)$. Sans encadrement.

Sur bol du service II.

3. XANTVS/FEC ou XAMTVS/FEC

Muette 66 (MML 89). Empreinte rectangulaire ( $10 \mathrm{x}$ 6). Encadrement de traits fins et nets. Ligature AM ou AN.

\section{XANTHVS et ZOILVS : O.C. 186}

\section{XANTHS OLI}

Muette 66 (MML 46). Empreinte rectangulaire (14 x $3,5)$ divisée en deux dans sa longueur par une barre verticale. Ligature ANTH.

Très probablement XANTHVS ZOILI.

Sur bols. Rare.

\section{ATTIVS : O.C. 205}

O.C. reproduit deux marques de cette série et les attribue à P. ATTIVS (205). Mais il n'y a à Lyon aucune marque P. ATTI alors qu'il existe de nombreuses C. ATTI. De même, O.C. attribue la marque HILARVS/ATTI à l'association HILARVS/(P) ATTI (212). Nous avons regroupé les deux séries de marques, ATTI et C. ATTI en admettant qu'il s'agit, à Lyon, du même potier. Nous avons écarté aussi la possibilité C. ATILIVS qui ne comprend qu'un scul T, alors qu'il est présent, avec deux L, à la Muette, sous la forme FLOS/C. ATILLI.

\section{ATTI}

Muette 66 . Empreinte rectangulaire $(14 \times 9)$. Sans encadrement.

Sur bols du service I et sur assiettes, plats. O.C. $205 \mathrm{~d}$ mais sur nos exemplaires A et $\mathrm{T}$ sont plus rapprochés.

2. ATTI

Muette 66 (MML 55). Empreinte rectangulaire (15 x 7). Sans encadrement.

Sur plats et assiettes. Non répertoriée dans O.C. 205.

\section{ATTI}

Muette 66. Empreinte $(12 \times 8)$. Encadrement.

Sur plats et assiettes; sur bols du service I (très abondante) et du service II (rare). Non reproduite dans O.C. 205.

\section{ATTI}

Muette 66. Même empreinte que la précédente mais avec deux points qui se reproduisent sur de nombreuses pièces : le premier sous la barre du $\mathrm{A}$, le second vers le milieu de la haste du T à droite. Ce n'est certainement pas accidentel et le poinçon est sûrement différent du précédent.

Sur plats, assiettes et bols (mais rare de façon générale).

\section{ATTI}

Muette 66. Empreinte rectangulaire (13 x 5). Sans encadrement.

Sur plats, assiettes et bols (services I et II). Abondante. Non reproduite dans O.C. 205.

\section{ATTI}

Muette 66. Fmpreinte rectangulaire plus ou moins losangée $(10 \times 6)$. Sans encadrement.

Sur plats, assiettes mais en majorité sur bols du service II. Abondante. Non reproduite dans O.C. 205.

7. 'TTA

Muette II (MML 107). Empreinte rectangulaire à coins arrondis $(8 \times 5)$. Sans encadrement. Il s'agit sûrement d'un ATTI inversé.

Sur plateau. Unique. Non répertoriée dans O.C.

8. C. ATTI

Muette 66. Empreinte rectangulaire très nette $(13 \mathrm{x}$ 8). Sans encadrement. Ligature AT. 
Sur bols uniquement et en majorité du service I. Non répertoriée dans O.C.

\section{C. ATTI}

Muette 66. Empreinte rectangulaire nette $(14 \times 6)$. Sans encadrement.

Sur bols uniquement, et en majorité du service I. Courante. Non répertoriée dans O.C.

\section{C. ATTI}

Muette 66 (MML 54). Empreinte rectangulaire (11 x 6). Sans encadrement.

Sur bols uniquement et en majorité du service II. Courante. Non répertoriée dans O.C.

\section{HILARVS/ATTI}

Muette 66 (MML 40). Empreinte rectangulaire (18 x 9). Sans encadrement.

Sur plats et assiettes uniquement. Abondante. O.C. 212.

\section{AVITTIVS ou AVTTIVS : non répertoriée dans O.C.}

\section{AVTTI}

Muette II. Empreinte rectangulaire mal délimitée (11 $x 5$ ). Sans encadrement.

Sur bols (2 ex.). Non répertoriée dans O.C.

\section{BASSUS : O.C. 323}

\section{BASSI}

Musée f.a. (EAD 7). Empreinte rectangulaire (14 x 5). Sans encadrement.

Sur bol.

\section{BRINNIVS : O.C. 335}

1. L. BRINNI (pl. 90, 4)

Musée f.a., R215 V38 (EAD 44).

\section{BUCCIO/VARI : voir VARI}

\section{CALAMVS : O.C. 359}

\section{CAI AM}

Muette 66. Empreinte circulaire légèrement ovalisée (diamètre : 8). Sans encadrement. Entre le début et la fin du nom, signe peu net, croissant ou pelte.

Sur bols du service II. Assez rare. O.C. 359b.

\section{CERDO : voir ANNI}

\section{C (H) RESTVS : O.C. 425}

\section{CRESTI}

Muette 66 (MML 179). Empreinte rectangulaire (11 x 3). Sans encadrement.

Sur bols du service II. Rare. O.C. 425 mais non déterminée avec précision.

\section{CLARVS : O.C. 443-444}

1. CLA (pl. 90,5)

Musée f.a. (EAD 69). Empreinte rectangulaire (env. $9 \times 5)$. Sans encadrement.

Sur assiette NI.

2. CLA (R) I (pl. 90, 6-7)

Haltern, $n^{\circ}$ 489-490 (HAL 134-135). Empreinte rectangulaire $(12 \times 9)$. Sans encadrement. Ligature AI. $n^{\circ} 489-490$ sur assiettes du service II.

3. CI ARI (pl. 90, 8)

Haltern, $n^{\circ} 495$ (HAL 136). Empreinte rectangulaire (15 x 8). Sans encadrement.

Sur assiette du service IC.

4. CLARI/ATEIAN (I) (pl. 90, 9)

Haltern, $n^{\circ} 497$ (HAL 137). Empreinte rectangulaire $(12 \times 8)$. Timbre imprimé sur deux lignes. Ligature AN. Encadrement.

Sur assiette du service II.

\section{COCI : non répertoriée dans O.C.}

1. COCI (pl. 90, 10)

Haltern, $n^{\circ} 870$ (HAL 185). Empreinte rectangulaire $(16 \times 8,5)$. Sans encadrement.

Sur assiette du service IC.

\section{COR : O.C. 467}

1. COR (pl. 90, 11)

Musée fa., Trion (EAD 38). Empreinte rectangulaire à angles arrondis (env. $9 \times 5$ ). Sans encadrement.

Sur bol du service II. 
DIOMEDES : O.C. 599

1. DIOM (pl. 90, 12)

Musée f.a., V312/12 (EAD 49). Empreinte rectangulaire $(17 \times 8)$. Encadrement.

Sur bol du service II ou Ha. 11.

2. DIOMEDES (pl. 90, 13)

Haltern, $n^{\circ} 518$ (HAL, 146). Empreinte rectangulaire ( $15 \times 6)$. Sans encadrement.

Sur assiette du service IC.

3. (...) NDIOI (pl. 90, 14)

Haltern, $n^{\circ} 511$ (HAI 145). Empreinte rectangulaire $(\vdots \mathrm{x} 4,5)$. Sans encadrement.

Sur FNI.

\section{FASTIDIENVS : O.C. 672}

1. C. FAS

Muette 66 (MML, 131). Empreinte rectangulaire (9 x 6). Sans encadrement. F archaïque.

Sur bols. Assez rare. O.C. 672 g2, f, i.

\section{FAVSTVS : O.C. 679}

\section{FAVS}

Muette 66 (MML 128). Empreinte rectangulaire (12 x 6). Sans encadrement. Ligature A V.

Sur bols. Rare. Non répertoriée dans O.C. 679.

\section{FELIX : O.C. 682, 684-685}

1. FELIX (pl. 90, 15)

Musée f.a., 31 (EAD 50). Empreinte rectangulaire $(15 \times 7)$. Sans encadrement.

Sur assiette NI.

2. FELI (X) (pl. 90, 16)

Haltern, $n^{\circ} 526$ (HAL 148). Empreinte rectangulaire (12 $\times 9)$. Sans encadrement.

Sur bol du service IC.

3. FELIX palme (pl. 90, 17)

Haltern, $n^{\circ} 528$ (IIAI, 150). Emprcinte rectangulaire (env. $15 \times 8$ ). Sans encadrement.

Sur bol du service II (?).
FIRMVS : O.C. 695

\section{FRMS/FEC:}

Muette 66 (MMI, 108). Empreinte rectangulaire $(8 \mathrm{x}$ $5)$. Encadrement de points très nets et plus ou moins réguliers.

Sur bol du service II. Unique. Non répertoriée dans O.C.

\section{FRM}

Muette 66 (MML 106). Empreinte vaguement rectangulaire $(9 \times 7)$. Sans encadrement. Marque caractérisée par un trait de soulignement barbelé, peut-être une palme.

Sur bols du service II. Asse\% rare. O.C. 695 d.

\section{FONTEIANVS : O.C. 703}

1. $\mathrm{FO}(\ldots)$

Muette 66 (MML 180). Deux lettres conservées, ligature FO, seulement. Très caractéristiques, elles permettent l'identification.

Un seul exemplaire. O.C. 703, 28.

2. FON (pl. 90, 18)

Haltern, $n^{\circ} 549$ (HAI 152). Empreinte rectangulaire (11 x 6). Sans encadrement.

Sur bol du service II.

3. FON (pl. 90, 19)

Musće f.a. (EAD 45). Empreinte circulaire (diam. 5).

Sur bol du service II.

\section{FRONTO : O.C. 713}

Marques caractérisées par une lettre $\mathrm{O}$ toujours nettement plus petite que les autres; toujours sur bols, essentiellement du service I et de petites dimensions.

\section{FRONTO}

Muette 66. Empreinte rectangulaire (11 x 5). Pas d'encadrement, mais sous les lettres légers tirets à droite, à gauche et au-dessus série de petites hachures plus ou moins distinctes selon les exemplaires. Ligature NT.

O.C. 7131 . 


\section{FRONT}

Muette 66. Empreinte rectangulaire $(9 \times 5)$. Sans encadrement. Ligature NT.

Non répertoriée dans O.C.

\section{FRONT}

Probablement la même empreinte que la précédente, mais ne reste de la ligature NT que les deux hastes du $\mathrm{N}$ et la partie gauche de la barre du $\mathrm{T}$. Le poinçon semble pourtant bien appliqué. S'agit-il de la mutilation du poinçon précédent?

Non répertoriée dans O.C.

\section{FRONIO/FECI}

Muette 66 . Empreinte rectangulaire $(9,5 \times 7)$. Sans encadrement. $\mathrm{F}$ archaïque.

O.C. $712 \mathrm{a}, \mathrm{d}$.

\section{FRONTO}

Muette 66. Empreinte allongée, côté gauche arrondi (11 x 5). Encadrement formé de deux traits seulement. Ligature NT.

O.C. $712 \mathrm{~g}, \mathrm{~h}, \mathrm{i}, \mathrm{n}$ ? Analyse MML. 78.

\section{FRONT}

Muette 66. Marque mutilée à droite. Empreinte rectangulaire. Sans encadrement. Ligature NT.

Unique. Non reproduite dans O.C. 713.

\section{GAMVS : O.C. 725}

Trois marques assez rares, toujours sur bols.

\section{GAMI}

Muette 66. Empreinte rectangulaire (11 x 5). Sans encadrement. Ligature AM.

Sur bols. O.C. $725 \mathrm{~d}, \mathrm{~m}, \mathrm{n}, \mathrm{q}, \mathrm{s}$, v.

\section{GAMI}

Muette 66 (MML 77). Empreinte rectangulaire (11 x 5,5). Sans encadrement.

Très comparable au poinçon précédent.

Sur bols. Non répertoriée dans O.C.

3. GAMI

Muette 66. Empreinte rectangulaire $(9 \times 6)$. Encadrement formé en haut et en bas de deux palmes aux extrémités gauches marquées d'un anneau. Ligature AM.

Sur bols. Assez rare. O.C. 725 e ? mais il y a des différences dans l'encadrement.

\section{SEMPRONIVS et L. GELLIVS : O.C. 747}

1. L. SEMPR./L. GELLI (pl. 90, 20-21) Musée f. a, R241 V17 (EAD 21), Haltern, n 568-570 (HAL 298, 154).

Empreinte rectangulaire à angles arrondis (env. $14 \mathrm{x}$ 8). Sans encadrement.

EAD 21 et $n^{\circ} 570$ sur bols du service $I, n^{\circ} 568$ sur assiette du service IB.

HA (?) : non répertoriée dans O.C.

1. HA

Muette 66 (MML 97). Assez grande marque dégradée. Empreinte rectangulaire ( $12 \times 7)$. Encadrement. Les deux grandes lettres sont précédées d'un signe difficile à interpréter.

Le catalogue de O.C. reproduit au n 144-408 a une estampille semblable mais lue ATEI, sans doute rétrograde. L'exemplaire de la Muette fait douter de cette lecture; même rétrograde, la ligature TEI en forme de H serait étonnante.

Sur assiette. Unique.

\section{INACHVS : O.C. 827}

\section{INACH}

Muette 66 (MML 187). Empreinte rectangulaire (12x 7). Encadrement formé à gauche de deux points, à droite de trois, en haut d'une guirlande (?) et en bas d'une palme. Ligature IN.

Grand bol du service I. Unique. O.C. 827 b.

\section{ICE OU ICV : non répertoriée dans O.C.}

Muette 66. Empreinte rectangulaire fortement imprimée $(7 \times 5)$. Encadrement rectangulaire.

Sur bols du service II. Assez abondante. Non répertoriée dans O.C. 


\section{IVCVNDVS : O.C. 835 ?}

1. IVCV palme (pl. 90, 22)

Musée f.a., 264 (EAD 64). Empreinte rectangulaire (? x 6). Sans encadrement.

Sur assiette NI.

\section{HILARVS/ATTI : voir ATTI}

\section{LESBIVS : non répertoriée dans O.C.}

\section{LESBI}

Muette 66 (MML 109). Empreinte rectangulaire aux angles arrondis $(8 \times 6)$. Encadrement rectangulaire très effacé à droite et à gauche.

Sur bols. Rare. Non répertoriée dans O.C.

\section{TITVS MALIVS FORTVNATVS : O.C. 942}

Marque imprimée exclusivement sur des bols du service II.

\section{TML/FORT/FECI}

Muette 66. Empreinte circulaire ovalisée (diamètre : 9 à 10). Sans encadrement.

FORT : $F$ archaïque,

FECI : $F$ archaïque.

Sur bols parfois de dimensions minuscules. Très abondante. O.C. 942, Vnn.

\section{FECIT/FORTV/TMAL}

Muette 66 (MML 56). Empreinte presque carrée $(8,5 \times 7,5)$. Sans encadrement.

FECIT : $F$ archaïque.

FORTV : F archaïque, V légèrement déplacé vers le haut.

Sur bols en général un peu plus grands que pour la graphie précédente. Moins abondante. O.C. 942, IV.

(N. NAEVIVS) HILARVS : O.C. 1084

\section{HIL AR}

Muette 66 (MML 79). Empreinte rectangulaire (15 x 7). Encadrement peu apparent, accusé aux quatre coins par des anneaux et formé de quatre palmes ou filets barbelés en arêtes de poisson. Ligature AR.

Sur grands bols. Très rare. O.C. 1084 e.

\section{HIIAAR}

Muette 66 (MML 26). Empreinte vigoureuse (14 x 6). Sans encadrement. Iigature AR.

Marque très semblable au $\mathrm{n}^{\circ}$ XIX 1 , la différence est dans l'absence d'encadrement.

Sur assiette. Unique. Peut-être O.C. 1084 e.

\section{NIGER : O.C. 1123 ?}

\section{NIGRI}

Muette 66. Empreinte rectangulaire $(10 \times 6,5)$. Sans encadrement.

Sur plats et assiettes. Très rare. Non répertoriée dans O.C. 1123.

\section{ATILLIVS ou (M. PVPIVS) FLOS ( ) F(ECIT ?) : O.C. 1204?}

Les marques assez abondantes sont toutes inscrites dans un encadrement dont la forme, malgré la variété des proportions, reste caractéristique : un rectangle dont les petits côtés sont convexes. Les lettres sont toujours penchées vers la droite et très soignées.

\section{FLOS/C. ATILLI}

Muette 66. Empreinte caractéristique de FLOS mais moins régulière ( $15 \times 6)$. Encadrement dont les petits côtés sont formés à gauche par une accolade, et à droite par une courbe convexe, gros points aux angles à droite.

Sur plats. Assez rare.

\section{FLOS}

Muette 66. Empreinte rectangulaire $(10 \times 6$ ). Encadrement formé d'un filet marqué aux angles par quatre points.

Sur plats (moutons). O.C. 1204 b.

\section{FLOS}

Muette 66 (MML 45). Empreinte rectangulaire $(6 \mathrm{x}$ $3,5)$. Encadrement.

Sur bol du service II. O.C. 1204 al? 


\section{QVADRATVS : O.C. 1460-1461}

1. QVADR

Muette 66. Empreinte rectangulaire (11 x 5,5), incomplet à droite et formé de trois palmes. H.l. 3. Ligatures VA, et DR.

Style de gravure très comparable à celui de la marque NIGER.

Sur plats. Rare. Non répertoriée dans O.C. 1460 et 1461 .

2. DRA

Muette 66 (MML 186). Marque mutilée à gauche. Empreinte probablement rectangulaire (hauteur : 7). Encadrement formé d'une palme en haut, d'anneaux reliés par une ligne ondulée en bas. Ligature DR.

Unique. O.C. 1460 ou 1461 mais sans la ligature DR.

\section{QVARTIO : O.C. 1463}

\section{QVARTIO}

Muette 66 (MML 96). Empreinte rectangulaire (12x 5). Encadrement rectangulaire. Ligature VA.

Sur plats. Rare. O.C. 1463.

\section{RASINIVS O.C. 1485}

\section{Marques rectangulaires}

\section{RASIN}

Muette 66. Empreinte rectangulaire, plus ou moins ovalisée $(13 \times 6)$. Encadrement.

Sur plats et assiettes. Très abondante. O.C. 1485, 199b ou 200.

\section{RASINI}

Muette 66. Empreinte nettement rectangulaire $(14,5 \mathrm{x}$ 7). Encadrement.

Sur assiette. Unique. Non reproduite dans O.C. au $n^{\circ} 1485$.

\section{RASINI}

Muette 66. Empreinte rectangulaire $\left(\begin{array}{lll}11 & \mathrm{x} & 5\end{array}\right)$. Encadrement de petits points nets et serrés.

Seule marque rectangulaire de Rasinivs sur bols. Asse\% abondante. Peut-être O.C. 1485, 197, 206, 207.

\section{RASIN}

Muette 66. Marque mutilée. Empreinte rectangulaire aux angles arrondis (hauteur : 6). Encadrement formé de points plus écartés et moins lisibles que sur l'exemplaire $n^{\circ}$ XXVII, 3.

Unique. Peut-être O.C. 1485, 197, 206, 207 comme pour le XXVII, 3.

\section{Marques circulaires}

\section{RASINI}

Muette 66. Empreinte circulaire nette et régulière (diamètre : 10). Sans encadrement, gros point en mamelon au centre.

Sur bols, en majorité du service I. Très abondante. O.C. $1485,184$.

\section{RASINI}

Muette 66. Empreinte circulaire nette (diamètre : 10). Sans encadrement et sans mamelon au centre.

Sur bols, en majorité du service I. Non reproduite dans O.C. 1485.

\section{RVFI RASI}

Muette 66 (MML 58). Empreinte rectangulaire $(10 \mathrm{x}$ 7). Encadrement incomplet; à droite et à gauche, trois anneaux reliés par un tiret. $\mathrm{F}$ archä̈que.

Sur bol à fond creux sur lequel la marque s'imprime mal et est rarement lisible dans sa totalité. O.C. 1543a, d, f.

8. ACASTI RAS (I) NI (pl. 90, 23)

Haltern, $n^{\circ} 676$ (HAL 163). Empreinte rectangulaire (17 x 9). Sans encadrement. Ligature IN. Palme sur les deux lignes.

Sur assiette NI. O.C. 1487

\section{RVSTICVS : O.C. 1619}

Marque imprimée exclusivement sur des bols et en majorité sur des bols du service II.

\section{RVSTICI}

Muette 66 (MML 57). Empreinte circulaire (diamètre : 9), pas de point central.

Très abondante. O.C. $1619 \mathrm{~g}$. 


\section{RVSTICI ( $\vdots)$}

Muctte 66. Empreinte circulaire (diamètre: 8 ). Sans encadrement, gros point central.

Assez rare. Non répertoriée dans O.C.

\section{SABINVS : O.C. 1645 ?}

1. SABII (pl. 90, 24)

Musée fa. (EAD 57). Empreinte rectangulaire (13 x 7). Encadrement.

Sur bol du service II.

\section{SENTIVS : O.C. 1730-1732}

Potier dont les marques sont les plus nombreuses et les plus variées à l'exception de Thyrsus qui pose un problème. Certaines sont soignées, d'autres négligées, mais elles ont toutes un style caractéristique. Elles sont rectangulaires sauf la dernière qui est d'ailleurs très rare. Il semble bien que la partic de l'atelier que nous avons fouillée était le centre de l'activité de Sentivs qui y a réalisé une grande quantité de sigillée lisse de formes très variées ainsi que de la sigillée décorée. L'ensemble des marques peut se décomposer en deux groupes : le groupe SENTI et le groupe C. SENTI.

\section{GROUPE SENTI}

\section{SENTI}

Muette 66. Grande empreinte rectangulaire (17 x 9). Sans encadrement. Ligature NTI.

Sur assiettes (très rare). Non répertoriée dans O.C.

\section{SENTI}

Muette 66. Empreinte rectangulaire (14 x 9). Sans encadrement. Ligature NTI du même type que le précédent.

Sur grands bols, en majorité du service I, rarissime sur plats et assiettes (2 ex.). O.C. 1729 tl, n2.

\section{SENTI}

Muctte 66. Empreinte rectangulaire $(13 \times 7)$. Encadrement de points très réguliers, serrés, presque confondus.

Sur bols en majorité du service II, rarissime sur plats et assiettes ( 1 ex). O.C. 1730, a, i.
4. SENTI

Muctte 66 (MMI. 52). Empreinte rectangulaire (13x 6,5). Sans encadrement. I.igature XTI du même type que le $n=1$.

Sur bols en majorité du service I, rare sur plats et assiettes. Non répertoriée dans O.C.

\section{SENTI}

Muette 66 . Empreinte rectangulaire (14 x 8). Sans encadrement. Ligature finale TI en forme de croix.

Sur bols et assiettes (très rare). Non répertoriée dans O.C.

6. SENTI

Muctte 66 . Empreinte rectangulaire $(13 \times 7)$. Sans encadrement. Ligature TI en forme de croix.

Sur bols en majorité du service I, rarissime sur assiettes (1 cx.).

7. SENTI

Muette 66 . Empreinte rectangulaire $(13 \times 6)$. Sans encadrement.

Sur bols (scrvice I) et assiettes. Très rare. Non répertoriée dans O.C.

\section{SENTI}

Muette 66. Empreinte aux angles arondis $(15 \times 8)$. Sans encadrement.

Sur assiettes. Très rare. Non reproduite dans O.C. 1730 .

\section{GROUPE C. SENTI}

\section{C. SENTI}

Muette 66. Empreinte rectangulaire (11 x 6). Sans encadrement. Marque caractérisée par la ligature ENTI avec l'habituelle ligature TI en forme de croix.

On trouve une fois cette marque répétée sur le pourtour extérieur d'un pied d'un calice sans doute de type Drag. 11. Elle figure aussi une fois en marque radiale.

Graphie apparaissant exclusivement sur des plats et assiettes. Tres abondante. O.C. 1732, 19, 78.

\section{C. SENT}

Muette 66. Empreinte rectangulaire (14x 7). Sans encadrement. Ligature NT. 
Graphie majoritaire sur plats et assiettes, rare sur bols (7 ex.). Non répertoriée dans O.C.

\section{C. SENT}

Muette 66 (MML 53). Empreinte rectangulaire (12 x 5). Sans encadrement. Ligature ENT inhabituelle.

Sur plateau. Unique. Non répertoriée dans O.C. 1732.

\section{C. SENTI}

Muette 66. Empreinte rectangulaire (12 x 5,5). Encadrement peu apparent mais complet, petits côtés obliques. Ligature ENTI.

Sur plats et assiettes. Rare (3 ex.). O.C. 1732, 83.

\section{C. SENT}

Muette 66 (MML 88). Empreinte rectangulaire (11 x 5). Encadrement très net. Ligature ENT.

Sur assiette. Unique. Non répertoriée dans O.C. 1732.

\section{C. SENT}

Muette 66. Empreinte mutilée et rectangulaire (hauteur : 8). Encadrement très net. Aucune ligature visible sur la partie conservée.

Sur assiette. Unique. Non reproduite dans O.C.

\section{C. SENTI}

Muette 66 (MML 83). Empreinte circulaire, la seule (diamètre : 8). Une palmette en forme de sapin occupe le centre. Iigature EN.

Sur petits bols du service II. Quatre exemplaires seulement (H.S.). O.C. 1732, 85b.

\section{SENTI ou SERVITI (?)}

Muette 66. Empreinte rectangulaire $(14 \times 8)$. Sans encadrement. Lettres dans le style du $n^{\circ}$ XXIX, 5.

Peut-on lire, avec la ligature ERV, SERVITI? Ou faut-il voir un $\mathrm{N}$ dont les deux premiers jambages ne se lieraient pas au troisième ; celui-ci serait barré dans le bas pour marquer la ligature TI, et lire SENTI? Nous préférons cette lecture, SENTI, à cause du style de la gravure.

Sur assiette du service I. Unique. Non répertoriée dans O.C.

17.C. SEN (T) I (pl. 90, 25)

Haltern, $n^{\circ} 714$ (HAL. 169). Empreinte rectangulaire (env. $9 \times 4$ ). Encadrement. Ligature TI.

Sur bol du service II. Unique.

\section{STABILIO : O.C. 1847}

\section{1. $\mathrm{STAB}$}

Muette 66 (MML 99). Empreinte vaguement rectangulaire $(10 \times 5,5)$.

Sur bol. Rare. O.C. 1847 a.

\section{TERTIVS : O.C. 1945 ?}

\section{TERTI}

Muette 75 (MML 197). Empreinte rectangulaire (17 x 7).

Sur assiette. Unique. Peut-être O.C. 1945 g2.

\section{TEVC : non répertoriée dans O.C.}

\section{TEVC}

Muette 66 (MML. 188). Empreinte (11 x 7). Sans encadrement, mais au-dessous, trois anneaux reliés par un trait fin.

Classée à la lettre $\mathrm{T}$ car son interprétation est difficile. S'agit-il plutôt de T. EVCARPVS? L'absence d'éléments de comparaison ne permet pas de trancher. Une seule référence dans Oswald, EVCARPVS potier de Trèves.

Sur assiette. Unique. Non répertoriée dans O.C.

\section{LVCIVS THYRSVS ET IOTHVR : O.C. 831, 2062, 2068}

\section{Premier groupe de huit marques}

Nous avions d'abord classé le groupe des estampilles traditionnellement lues IOTHVR, à la lettre I. Dans ce groupe, nous avions rassemblé toutes les marques présentant un V. Elles ont toutes la ligature TH et VR. Cinq de ces huit marques ont une première lettre qui peut être interprétée comme un I. Toutes ont un point entre I ou L et $\mathrm{T}$, qui peut grossir jusqu'à ressembler à un $\mathrm{O}$ de petite taille (comme ceux de FRONTO).

Loeschcke, qui en a relevé un certain nombre à Haltern, a admis la lecture IOTHVR, par analogie avec un nom gravé sur une fibule de Ia Tène. Il en est de même dans O.C., mais la découverte dans le même atelier des groupes IOTHVR et THYRSVS, côte à côte, nous a amené à nous demander s'ils n'appartenaient pas à la même firme. 


\section{Deuxième groupe de douze marques}

Il est caractérisé par le Y, la première lettre qui est toujours $\mathrm{L}$, la présence de une ou deux lettres de plus en finale : $\mathrm{S}$ et I dans dix marques sur douze, la disparition du $\mathrm{H}$ dans six cas sur douze.

La lecture THYRSVS est absolument certaine. Entre les deux groupes, un certain nombre de différences ne sont pas très probantes : V et $Y$ sont confondus en général dans les estampilles.

L'absence de S ou SI en finale dans le premier groupe peut être pris pour une abréviation.

Dans le premier groupe, on peut dire que le $\mathrm{O}$ cntre I et $\mathrm{T}$ diminue jusqu'à devenir semblable à un point, mais le raisonnement inverse peut être tenu : c'est le point qui grossit.

En ce qui concerne la première lettre I ou L, il y a peut-être eu confusion du graveur.

Un certain nombre de ressemblances sont à noter : les marques $6,7,8$ du premier groupe sont intermédiaires entre les deux groupes. Comme l'ensemble du groupe 1, elles ont un V et pas de terminaison S ou SI. Mais, comme la plupart des marques du groupe 2, elles ont un L et seulement un point entre $\mathrm{L}$ et $\mathrm{T}$.

Dans le premier comme dans la moitié du deuxième groupe, les ligatures TH et VR sont les mêmes.

Il paraît difficile de trancher pour l'instant de façon définitive. Nous considérons provisoirement que ces marques appartiennent au même potier L. THYRSVS ou à la même firme et nous les avons regroupées pour faciliter les comparaisons. Nous reprendrons plus tard ce problème et une solution nous sera peut-être apportée par l'étude des zones de diffusion.

\section{GROUPE IOTHVR}

\section{Série IOTHVR}

Lettre $\mathrm{O}$ toujours plus petite, parfois réduite à un point.

\section{IOTHVR}

Muettc 66. Empreinte rectangulaire (12 x 6,5). Sans encadrement. Ligatures TH et VR.

Sur assiette. O.C. 831 h.

\section{IOTHVR}

Muette 66. Empreinte rectangulaire (11,5 x 6). Encadrement formé de points minuscules. Ligatures TH et VR.

Sur assiettes. O.C. 831, e, h.

\section{IOTHVR}

Muette 66. Empreinte aux petits côtés obliques $(10 \mathrm{x}$ $6,5)$. Encadrement très fin peu visible à gauche. Ligatures TH et VR.

Sur assiettes et bols. Non reproduite dans O.C. 831 .

\section{IOTHVR}

Muette 66. Empreinte vaguement rectangulaire $(8 \mathrm{x}$ 5). Encadrement très fin, peu visible. Ligatures TH et VR.

Graphie apparaissant exclusivement sur des bols en majorité du service I ( 2 ex. sur service II). Non reproduite dans O.C. 831 .

\section{IOTHVR}

Muette 66. Empreinte rectangulaire (5,5 x 4), la plus petite du groupe. Sans encadrement. H.1. 2,5. I igatures TH et VR.

Sur assiettes et bols. O.C. 831 , d, h.

\section{Série L. THVR}

Sur ces marques, la première lettre est un L suivi d'un point. Les lettres THVR sont groupées en deux ligatures comme dans la série précédente.

\section{I. THVR}

Muette 66 (MML 74). Empreinte à peu près rectangulaire (13 x 8). Sans encadrement. Ligature TH et VR. Sur bol du service I. Unique. Non répertoriée dans O.C. 2066.

\section{L. THVR}

Muette 66. Empreinte rectangulaire (11,5 x 7). Sans encadrement. Ligature TH et VR.

Sur bols. O.C. 2062, 54, 59 ? Identification sous réserve car O.C. ne donne qu'une lecture.

\section{LOTHVR}

Muette 66. Empreinte très vaguement rectangulaire ( $7 \times 5$ ). Sans encadrement. Ligatures TH et VR.

Graphie apparaissant exclusivement sur des bols, en majorité du service I O.C. 2062, 18, 19, 24a, d 30, 53. 


\section{GROUPE L. THYRSVS}

\section{Série L. THYRSI avec $H$}

\section{L. THYRSI}

Muette 66. Empreinte rectangulaire (21 x 7). Sans encadrement.

Aucune ligature.

Sur grand plateau. Unique. Non reproduite dans O.C. 2062.

\section{L. THYRSI}

Muette 66. Empreinte rectangulaire irrégulière $(13 \mathrm{x}$ 6), fond strié en oblique. Sans encadrement. Ligature TH et ligature caractéristique YR.

Graphie apparaissant exclusivement sur bols du service I (8 ex.). O.C. $2068,38$.

\section{L. THYRS}

Muette 66. Empreinte rectangulaire mal délimitée à droite (12 x 6). Sans encadrement.

Marque identique à la précédente mais sans I final. S'agit-il d'une altération ou d'une mauvaise impression du poinçon $n^{\circ} 10$ ? Le fait qu'elle soit fréquente semble prouver qu'il s'agit bien d'une altération.

Sur bols. Non reproduite dans O.C.

\section{L. THYR}

Muctte 66. Empreinte rectangulaire côté droit mal délimité $(11 \times 6)$. Sans encadrement.

Même marque que le $n^{\circ} 10$ mais l'altération affecte les lettres S et I.

Sur bol. Unique. Non reproduite dans O.C. 2062.

\section{I. THYRSI}

Muette 66. Empreinte rectangulaire très nette $(11 \mathrm{x}$ 6). Sans encadrement. Ligatures TH et YR.

Graphie apparaissant exclusivement sur bols du service I (5 ex.). Assez courante. Non répertoriée dans O.C.

\section{Série L. TYRS sans H}

\section{L.T.Y.RS}

Muette 66. Empreinte rectangulaire $(14 \times 6)$. Sans encadrement.

Graphie apparaissant exclusivement sur plats et assiettes. Non reproduite dans O.C. au n ${ }^{\circ} 2062$.

\section{L.T.YRS}

Muette 66. Empreinte rectangulaire (14 x 6). Sans encadrement.

Marque identique à la précédente mais le point entre le Y et le R a disparu.

Graphie apparaissant exclusivement sur plats et assiettes. Assez rare. Non reproduite dans O.C. 2062.

16. L. TYRS

Muette 66. Empreinte rectangulaire ( $14 \times 6$ ). Sans encadrement.

Marques identiques aux $\mathrm{n}^{\circ} 14$ et 15 mais il y a un seul point entre $\mathbf{L}$. et $\mathbf{T}$.

Graphie apparaissant exclusivement sur plats et assiettes. Non reproduite dans O.C. 2062.

\section{L. TYRS}

Muette 66. Empreinte rectangulaire (13 x 7). Encadrement formé de quatre traits non jointifs.

Graphie apparaissant exclusivement sur plats et assiettes. O.C. 2062, 42.

\section{LTYRS}

Muette 66. Empreinte irrégulière $(14 \times 5)$. Encadrement incomplet formé en haut d'une ligne en zigzag peu lisible, sur les trois autres côtés peut-être quelques points irréguliers non alignés et très peu visibles.

Sur assiette. Unique. Non reproduite dans O.C. 2062.

\section{L. TYRS}

Muette 66. Empreinte rectangulaire $(9 \times 6)$. Encadrement très net.

Sur bols du service II ( 3 ex.). Non reproduite dans O.C. 2062.

\section{L. TYRS}

Muctte 66 (MMI 178). Empreinte rectangulaire très nette $(14 \times 7)$.

Non reproduite dans O.C. 2062.

\section{TIGRANVS : O.C. 1995}

Séric de marques rectangulaires toujours disposées sur deux lignes et souvent dans un encadrement plus ou moins élaboré. Cette série se compose de deux groupes: un groupe avec finale NI et l'autre NEI. Nous n'expliquons pas pour l'instant cette anomalie. 
S'agit-il seulement d'une fantaisie de graveur? L'appartenance à la même firme ou au même potier est indiscutable. Notons d'ailleurs que pour le groupe TIGRANI (graphies 1 à 3), la coupure en deux lignes s'opère entre les lettres $\mathrm{G}$ et $\mathrm{R}$ et pour TIGRANEI entre R et A (graphies 4 à 6 ).

\section{C. TIG/RANI}

Muette 66. Empreinte rectangulaire à angles arrondis $(10 \times 8)$. Encadrement à trois côtés seulement : à gauche, tirets obliques peu nets et plus apparents vers le haut, c'est peut-être la trace d'une ligne ondulée comme dans O.C. 1195,11 ; à droite une palme et en bas un trait.

Graphie apparaissant exclusivement sur des bols, en majorité du service I. Abondante. Peut-être O.C. 1995, 11 ?

\section{C. TIG/RANI}

Muette 66. Fmpreinte rectangulaire aux angles arrondis $(10 \times 9)$. Encadrement formé pour les petits côtés par deux lignes ondulées plus visibles à droite, qui se recoupent en formant des anneaux allongés. Ligature NI.

Graphie apparaissant exclusivement sur des bols? Très rare. (H.S.) O.C. 1995, 10.

\section{C. TIG/RANI}

Muette 66. Empreinte rectangulaire (12 x 9). Encadrement formé de traits non jointifs aux angles; celui de droite est traité en palme, dentelé seulement à gauche. Même disposition qu'au $n^{\circ} 2$ arec les anneaux de séparation.

Graphie apparaissant exclusivement sur des bols? Très rare. (H.S.). Non répertoriée dans ().C. 1995.

\section{GROUPE C. TIGRANEI}

\section{C. TIGR/ANEI}

Muette 66. Empreinte rectangulaire $(13 \times 7,5)$. Encadrement très net.

Sur assiettes. Très rare (H.S.). O.C. 1995, 26.

\section{C TIGR/ANEI}

Muettc 66 (MML 87). Empreinte rectangulaire (12 x 7). Encadrement réduit à un trait à gauche.

Sur assiettes et sur bols. Rare. O.C. 1995, 13?

\section{C TIGR/ANEI}

Muette 66. Empreinte rectangulaire incomplète à gauche (hauteur : 7). Pas d'encadrement visible.

On peut se demander s'il ne s'agit pas d'une altération ou d'une mauvaise impression du $n^{\circ} 5$.

O.C. 1995, 13?

\section{TTA : voir ATTI}

\section{TVLLIVS : non répertoriée dans O.C.}

Marque apparaissant exclusivement sur des bols.

\section{M. TVLI}

Muette 66. Empreinte rectangulaire $(13 \times 6)$. Sans encadrement.

Sur bols en majorité du service I. Abondante. Non répertoriée dans O.C.

2. TVI.

Muctte 66. Empreinte rectangulaire $(11 \times 5,5)$. Sans encadrement.

\section{SEX. VARIVS : O.C. 2235-2237}

\section{VARI}

Muette 66 (MML 90). Empreinte rectangulaire $6 \mathrm{x}$ $4,5)$. Encadrement de traits très fins et nets. Ligature VA.

Sur petit bol. O.C. 2234 bc.

\section{SEXT. VARI}

Muette 66. Empreinte rectangulaire allongée et étroite $(15 \times 4,5)$. Ligature VAR.

Sur bols. Rare. O.C. $2235 \mathrm{~g} 2$.

3. SEXTI/VARI (pl. 90, 26)

Musée fa., 205 (EAD 56). Empreinte rectangulaire (env. 11 x 5). Timbre imprimé sur deux lignes. Sans encadrement.

Sur bol du service II.

\section{BVCCIO/VARI FEC}

Muette 66 (MML 51). Empreinte rectangulaire (14 x 9) toujours remarquable par sa netteté. Timbre imprimé sur deux lignes. Ligature VA.

Sur bols. Rare. O.C. 2237. 


\section{VILLIVS : O.C. 2372}

1. VILL (pl. 90, 27) Musée f.a. (EAD 9). Empreinte rectangulaire à bords dentelés $(13 \times 5)$. Sans encadrement. Sur plat NI.

2. VIIL (pl. 90, 28) Musée f.a. (EAD 55). Empreinte rectangulaire (11 x 6). Encadrement haut/bas.

Sur bol du service II.

\section{VMBRICIVS : O.C. 2385}

\section{VMBRICIVS}

Muette 66 (MML 102). Empreinte rectangulaire (18x $8)$. Encadrement formé de traits.

Sur grand plateau. Unique. Non répertoriée dans O.C.

\section{VRBANVS : O.C. 2498}

\section{VRBANI}

Muette 66 (MML 110). Empreinte rectangulaire $(14,5 \times 6)$. Encadrement très net de traits fins. Ligature VR.

Sur bol. Unique. O.C. 2498 bl.

2. VRBANV (pl. 90, 29)

Haltern, $n^{\circ} 862$ (HAL 179). Empreinte rectangulaire à grands côtés convexes (dimensions maximales $17 \mathrm{x}$ 10). Encadrement double. Ligature AN.

Sur bol du service II (?).

3. VRBANV (S) /A (...)V (pl. 90, 30)

Haltern, $n^{\circ} 863$ (HAL 180). Empreinte rectangulaire (14 x 9). Encadrement. Timbre imprimé sur deux lignes. Ligature AN.

Sur assiette du service II.

\section{MARQUES DE LECTURE CONJECTURALE}

1. AMA? (pl. 90, 31)

Haltern, $n^{\circ} 902$ (HAL 189). Empreinte rectangulaire (14 x 7). Sans encadrement.

Sur assiette du service IC.
2. ITVTN (pl. 90, 32)

Haltern, $\mathrm{n}^{\circ} 878$ (HAL 187). Empreinte rectangulaire (env. $16 \times 5$ ). Sans encadrement.

Sur bol du service $\mathrm{I}$.

3. REI (pl. 90, 33)

Haltern, $n^{\circ} 879$ (HAL 188). Empreinte rectangulaire (env. $8 \times 5$ ). Sans encadrement. Marque rétrograde. Sur bol (service II ?).

4. TIT. ROR (pl. 90, 34)

Musée f.a., V312 12 (EAD 48). Empreinte rectangulaire $(13 \times 6)$.

Sur bol du service II.

5. (...) T I F (pl. 90, 35)

Haltern, $n^{\circ} 873$ (HAL 315). Empreinte rectangulaire (? $\times 4)$.

Sur assiette du service IB.

\section{VLON ou ELON}

Muette 66. Empreinte irrégulière $(12 \times 6)$. H.l. 3. On peut lire $\mathrm{E}$ (archaiqque), L, O, N ; le premier jambage du $\mathrm{N}$ est toujours cassé en haut, la marque est barrée en haut par un trait horizontal cassé vers la gauche, tous les exemplaires portent cette particularité.

Marque apparaissant exclusivement sur des bols, en majorité du service I (un seul exemplaire sur service II). Non répertoriée dans O.C.

7. Marque rectangulaire. Elle résulte sans doute d'une mauvaise impression d'un poinçon que nous n'avons pas pu identifier : peut-être SENTI ${ }^{\circ}$ XXIX, 12. Elle évoque peut-être aussi d'autres variantes de SENTI qui n'existent pas à la Muette.

8. MML 101. Empreinte rectangulaire $(8 \times 6)$. Encadrement fortement pointé aux angles et peutêtre divisé par une barre verticale, le côté gauche porte trois points en ergot, la barre du centre porte aussi trois ergots : EDEMI ou EDEVI, DV ou DM. Sur petits bols. Deux exemplaires seulement.

9. Empreinte arrondie $(10,5 \times 5)$. Encadrement formé de deux lignes horizontales nettement marquées. Ressemble au FRONTO XVI, 5 dont seuls les caractères du milieu auraient été imprimés. Il ne s'agit sûrement pas d'un accident puisque ce phénomène est 
observé sur sept exemplaires. Nous ne proposons pas de lecture.

Non reproduite dans O.C.

10. MML 111. Muette II. Empreinte déformée (13 x 6). Il s'agit peut-être d'une estampille rétrograde. En choisissant cette solution, nous proposons pour les trois derniers signes de droite à gauche, deux T liés et I. Le premier signe à droite peut être un $\mathrm{N}$ ou un A lié à un autre signe. Peut-on lire ATTI rétrograde? Cette lecture ne nous satisfait pas.

Unique. Non reproduite dans O.C.

11. MML 184. Marque à peu près illisible sans doute à cause de l'usure du poinçon qui est cependant fortement imprimé. Petite empreinte aux angles arrondis $(11 \times 5)$. On devine seulement trois lettres au centre : $\mathrm{S}$ et $\mathrm{E}$ à peu près sûrement, on pourrait ensuite lire I, T ou N.

Unique. Non reproduite dans O.C.

\section{ESTAMPILLES ANÉPIGRAPHES}

1. Empreinte circulaire (diamètre : 9). Rouelle de seize rayons qui ne touchent pas l'anneau central.

Sur petits bols. Rare. Non reproduite dans O.C.

2. MML 103. Empreinte circulaire (diamètre : 15). Rosette de onze points creux peu réguliers, encadrés à l'extérieur par un sillon circulaire et entourant un anneau à gros point central. Il ne s'agit pas d'un poinçon mais d'une marque composée à main levée avec l'extrémité d'un stylet.

Sur bol. Rare.

\section{LES MARQUES SUR IMITATIONS DE SIGILLÉE}

\section{GROUPE LYON}

Dix timbres représentent huit marques différentes (tabl. XIII).

1. ARRE (TINVM $\vdots$ ) : graphie ARRE (pl. 91, 1)

Musée f.a. Loyasse, EAD 22. Empreinte rectangulaire à angles arrondis $(10 \times 7)$. Encadrement haut/bas.

Timbre central sur bol hémisphérique à vernis brun.
2. AVL (VS) : graphie?

Musée f.a. Citerne R.R., SRG; 101 et 107

3. DIO (?) : graphie/IO (pl. 91, 4)

Lyon, Verbe-Incarné, LSS 56. Empreinte rectangulaire (env. 12 x 7). Sans encadrement. La première lettre est un delta.

Timbre central sur petit bol à bord oblique à vernis rouge.

4. P. HERTORIVS : graphie PHER (pl. 91, 5)

Lyon, Verbe-Incarné. Empreinte rectangulaire (env. $12 \times 8$ ). Encadrement droite/gauche.

Timbre sur FNI à vernis rouge.

5. PAMPHILVS : graphie PAMPHILVS FECIT (pl. 91,6) Musée f.a., EAD 29. Empreinte rectangulaire (12 x 9). Sans encadrement. Nom imprimé sur trois lignes séparées par des traits horizontaux.

Timbre central sur assiette NI à vernis rouge.

6. PRIMVS : graphies PRI (Verbe-Incarné) et PR (I) M (Farges) (pl. 91, 7-8)

Lyon, Verbe-Incarné, EAD 76. Empreinte rectangulaire à angles arrondis (11 x 7). Sans encadrement. Timbre central sur assiette à vernis rouge de type proto IB.

Rue des Farges, EAD 73. Empreinte rectangulaire $(11 \times 8)$. Sans encadrement. Timbre central sur bol à vernis rouge de type proto IB.

7. A VIBIVS SCROFVLA : graphie A (...) / SCROF (pl. 91, 9)

Lyon, Verbe-Incarné, ISS 42. Empreinte rectangulaire (13 $x$ ?). Sans encadrement. Nom imprimé sur deux lignes séparées par un trait horizontal.

Timbre central sur assiette NI à vernis rouge.

8. THYRSVS : graphie?

Musée f.a. Citerne R.R., SRG 106.

\section{GROUPE VIENNE}

Sept timbres représentent six marques différentes (tabl. XIV).

1. ALB (..) : graphie?

Musée f.a. Citerne R.R., SRG 103. 
2. AM (ANDVS) FEC (IT) : graphie AM/FEC (pl. 91, l 1) Saint-Romain-en-Gal, SRG 65/69. Empreinte rectangulaire $(11 \times 12)$. Encadrement. Nom imprimé sur deux lignes; ligature AM, FEC rétrograde.

Timbre central sur vase NI à vernis brun.

3. ARGE (NTILVS) F (ECIT) : graphie ARGEF (pl. 91, 12)

Saint-Romain-en-Gal, SRG 61 et 105. Empreintes rectangulaires (? x 11;23 x 11). Encadrements.

Timbres centraux : $n^{\circ} 61$ sur plat à bord oblique à vernis brun, $n^{\circ} 105$ sur FNI à vernis rouge.

4. CELAR (IVS?) : graphie CELAR (pl. 91, 13) Saint-Romain-en-Gal, SRG 62. Empreinte rectangu- laire à angles arrondis (env. 21 x 10). Sans encadrement. Ligature AR.

Timbre central sur FNI à vernis rouge.

5. IVLIV (S) : graphie IVI.IV (pl. 91, 14)

Lyon, Cybèle, 1975, SRG 108. Empreinte rectangulaire ( $15 \times 7)$. Encadrement haut/bas. Timbre central sur bol à parois obliques à vernis rouge.

6. SEXTVS VALERIVS NIGER : graphie SEX VAL/NIGER (pl. 91, 15)

Saint-Romain-en-Gal, SRG 64. Empreinte rectangulaire $(7 \times 6)$. Nom imprimé sur deux lignes et encadré de traits horizontaux.

Timbre central sur bol du service II.

M. G., J. I., M. P.

Tabl. XIII. Liste des marques sur imitations de sigillée, groupe Lyon.

\begin{tabular}{|l|l|l|l|l|}
\hline $\mathbf{n}^{\circ}$ analyse & \multicolumn{1}{|c|}{ provenance } & \multicolumn{1}{c|}{ graphie } & \multicolumn{1}{c|}{ marque } & \multicolumn{1}{c|}{ forme } \\
\hline EAD 22 & Musée f.a. (Loyasse) & ARRE & ARRE(TINV'M?) & bol \\
\hline SRG 101 & Musée f.a. (citerne RR) & AVLI & AVLVS & indéterminée \\
\hline SRG 107 & Musée f.a. (citerne RR) & AVLI & AVLVS & indéterminée \\
\hline LSS 56 & Lyon, Verbe-Incarné & IO & DIO? & bol à bord oblique \\
\hline LSS 62 & Lyon, Verbe-Incarné & P(H)ER & P.HERTORIVS & FNI \\
\hline EAD 29 & Musée f.a. & PAMP/HILVS/FECIT & PAMPHILVS & assiette NI \\
\hline EAD 73 & Lyon, Farges & PR(I)M & PRIMVS & bol service IB précoce \\
\hline EAD 76 & Lyon, Verbe-Incarné & PRI & PRIMVS & assiette service IB précoce \\
\hline LSS 42 & Lyon, Verbe-Incarné & A(...)/SCROF. & A VIBIVS SCROFVLA & assiette NI \\
\hline SRG 106 & Musée f.a. (citerne RR) & $?$ & THYRSVS & indéterminée \\
\hline
\end{tabular}

Tabl. XIV. Iiste des marques sur imilations de sigillée, groupe Viemne.

\begin{tabular}{|c|c|c|c|c|}
\hline $\mathrm{n}^{\circ}$ analyse & provenance & graphie & marque & forme \\
\hline SRG 103 & Musée f.a. (citerne RR) & ALB & ALB(ANVS)? & indéterminée \\
\hline SRG 65/69 & Saint-Romain-en-Gal & AMF & AM(ANDVS;/FEC(IT) & indéterminée \\
\hline SRG 105 & Saint-Romain-en-Gal & ARGEF & ARGE(NTILV'S) F(ECIT) & plat à bord oblique \\
\hline SRG 61 & Saint-Romain-en-Gal & ARGEF & ARGE(NTILV'S) F(ECIT) & indéterminée \\
\hline SRG 62 & Saint-Romain-en-Gal & CELAR & CELAR(IVS)? & indéterminée \\
\hline SRG 108 & Lyon, Cybèle 1975 & IVLIV & IVLIV(S) & bol à bord oblique \\
\hline SRG 64 & Saint-Romain-en-Gal & SEX VAL / NIGER & SEX(TVS) VAL(ERIVS) / NIGER & bol service II \\
\hline
\end{tabular}




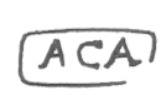

1

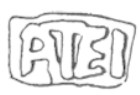

6

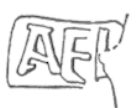

11

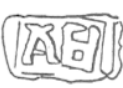

16

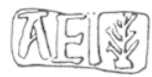

21

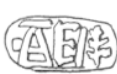

26

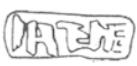

31

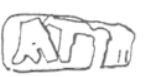

36
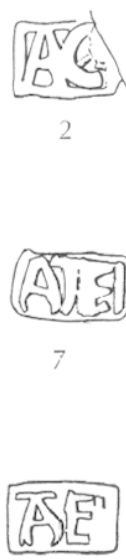

12

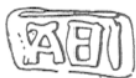

17

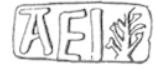

22
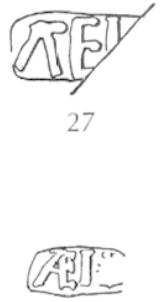

32

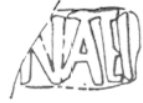

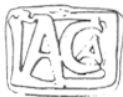

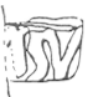

4

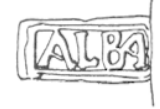

5
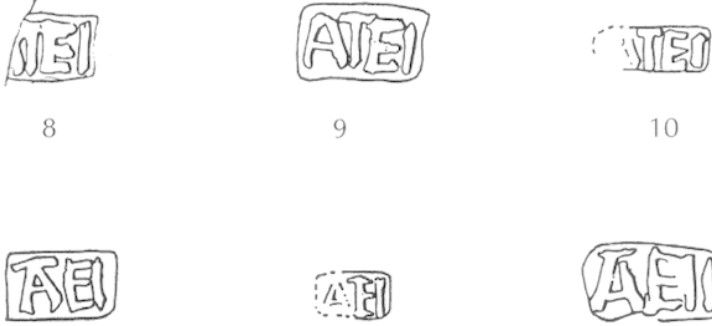

14

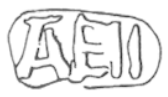

15
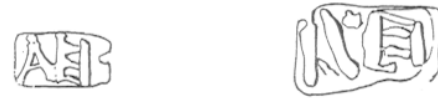

19

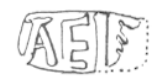

20
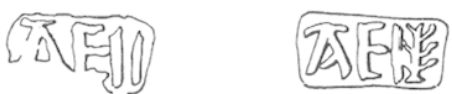

24

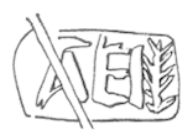

25

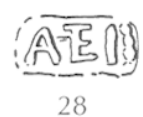

A

29

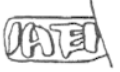

30
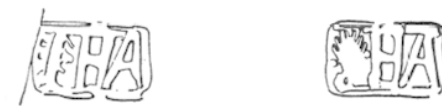

0

33

34

35

Pl. 89. Sigillée, marques et graphies lyonnaises non attestées sur l'atelier (2-37, d'après von Schnurbein, 1982) (échelle: 1/1). 


\begin{tabular}{|c|c|c|c|c|}
\hline HEVODI & XANTII & $\overline{B A} S S D$ & L.BRINNIVS & C(A) \\
\hline 1 & 2 & 3 & 4 & 5 \\
\hline & 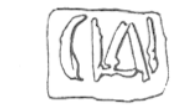 & & 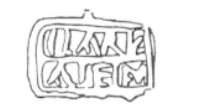 & $\begin{array}{l}28 \sqrt{212} \\
052,50\end{array}$ \\
\hline 6 & 7 & 8 & 9 & 10 \\
\hline $\mathrm{COP}$ & DTOM & (ब) $(0) 2$ & (4) & FE【X \\
\hline 11 & 12 & 13 & 14 & 15 \\
\hline$\frac{\text { (555) }}{(05595)}$ & 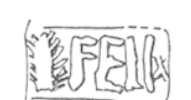 & $(\overline{P O})^{\circ}$ & $1 / 5$ & $\begin{array}{l}\text { L.SEMPR } \\
\text { 1.CELLL }\end{array}$ \\
\hline 16 & 17 & 18 & 19 & 20 \\
\hline 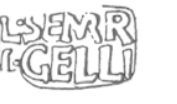 & IVCV草 & 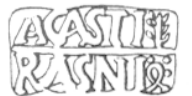 & $5 A B D$ & खुत्व \\
\hline 21 & 22 & 23 & 24 & 25 \\
\hline $\begin{array}{l}\text { SEXPT } \\
\text { SAARI }\end{array}$ & VII & , प्रLL & 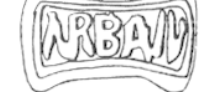 & 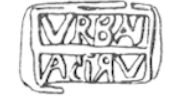 \\
\hline 26 & 27 & 28 & 29 & 30 \\
\hline$\Delta \nabla \Delta \Delta \nabla \Delta \nabla_{1}^{\prime}$ & ardurses & (BD25) & TITROR & (3) \\
\hline 31 & 32 & 33 & 34 & 35 \\
\hline
\end{tabular}

Pl. 90. Sigillée, marques el graphies lyonnaises non altestées sur l'atelier (6-10,13, 14, 16-18, 21, 23, 25, 29-33, 35, d'après von Schnurbein, 1982) (échelle : $1 / 1$ ). 


\section{ARR}

1

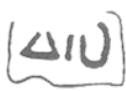

4

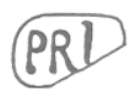

7

\section{ALBANVS}

10

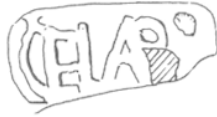

13
AVLI
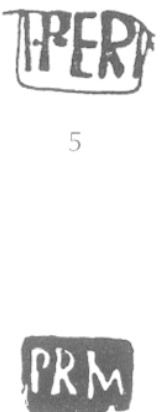

8

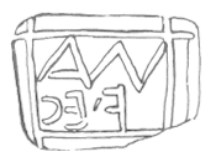

11

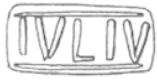

14
AVLI

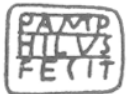

6

$\left(\frac{A}{(R \cup F)}\right)$

9

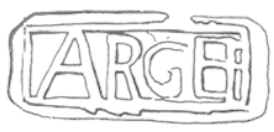

12

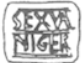

15

P1. 91. Imitations de sigillée. timbres homnais al viennois téchelle: $l / l$ ). 